\title{
Value of pretreatment MRI determined parameters for predicting outcome after radio- frequency ablation of hepatocellular carcinoma
}

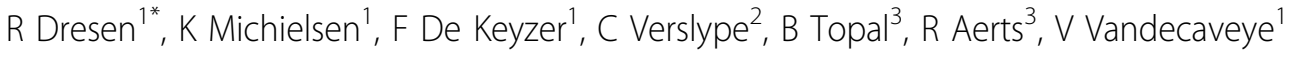 \\ From International Cancer Imaging Society Meeting and 15th Annual Teaching Course (ICIS 2015) \\ London, UK. 5-7 October 2015
}

\begin{abstract}
Aim
To evaluate whether pretreatment magnetic resonance imaging (MRI) determined imaging parameters are predictive for outcome in hepatocellular carcinoma (HCC) treated with radio-frequency ablation (RFA).
\end{abstract}

\section{Methods}

Thirty-seven patients with HCC treated by RFA were evaluated. Lesion number, size and segmental location, T2-weighted $(\mathrm{w})$, arterial, portal-venous and venous contrast-phase, b600 diffusion-w imaging (DWI) and delayed phase contrast-enhanced imaging pattern were assessed at MRI. The separate imaging patterns as well as pretreatment clinical variables were correlated with outcome (disease free survival longer or shorter than 1 year) using a chi-square test with multiple variables and Mann-Whitney U test respectively. Pretreatment clinical variables and imaging parameters were correlated with Keratin 19 and microvascular invasion status at the biopsy during RFA.

\section{Results}

None of the pretreatment patient- or tumour-related parameters correlated to disease free survival ( $p>0.5)$.

The portal-venous, venous phase and b600 DWI imaging pattern showed strongest correlation with disease free survival $(\mathrm{p}=0.00023, \mathrm{p}=0.00003$ and $\mathrm{p}=0.0002$ respectively). Also correlation was found for T2w imaging pattern $(\mathrm{p}=0.007)$, and hepatobiliary phase imaging pattern $(\mathrm{p}=0.017)$. Patients with tumour recurrence within 1 year $(n=14)$ showed persistent venous rim- or nodular enhancement in 13 patients and b600 DWI rim-like hyperintensity in 9 patients correlating with microvascular invasion at biopsy $(\mathrm{p}=0.04)$. Patients disease free for at least 1 year $(n=23)$ showed venous washout in 22 of 23 patients and whole-lesion hyperintensity b600 DWI in 18 patients.

\section{Conclusion}

Pretreatment venous rim-enhancement and rim-like intensity at b600 DWI were strongest predictors of treatment failure within the first year after RFA of HCC.

\section{Authors' details}

${ }^{1}$ Department of Radiology, Leuven Cancer Institute, University Hospitals Leuven, KU Leuven, Leuven, Belgium. ²Department of Hepatology, Leuven Cancer Institute, University Hospitals Leuven, KU Leuven, Leuven, Belgium. ${ }^{3}$ Department of Surgery, Leuven Cancer Institute, University Hospitals Leuven, KU Leuven, Leuven, Belgium.

Published: 2 October 2015

* Correspondence: elleke.dresen@uzleuven.be

'Department of Radiology, Leuven Cancer Institute, University Hospitals

Leuven, KU Leuven, Leuven, Belgium

Full list of author information is available at the end of the article 